\title{
Genic and karyotypic selection on an inversion polymorphism in the seaweed fly, Coelopa frigida
}

\author{
R. K. Butlin* and \\ T. H. Day
}

\author{
Department of Genetics, University of Nottingham, \\ University Park; Nottingham, NG7 2RD
}

\begin{abstract}
The seaweed fly, Coelopa frigida, is polymorphic for an inversion on chromosome I. Heterozygotes show higher viability than either homozygote. A series of crosses between lines derived from two geographically distant populations indicates that a significant component of selection is due to the deleterious effects of recessive alleles. Selection at the karyotypic level, if present, is relatively weak.
\end{abstract}

\section{INTRODUCTION}

Chromosomal inversion polymorphisms are often subject to strong selection pressures despite the fact that the only direct effects of the inversion are a change in gene order, and suppression of recombination in heterozygotes (Dobzhansky, 1971). Two types of explanation have been proposed to account for this selection. The two sequences may accumulate different sets of mutations that have recessive deleterious effects, or are overdominant such that structural homozygotes will, on average, be less fit than heterozygotes (Sturtevant and Mather, 1938; Ohta, 1971). Alternatively, the suppression of recombination may hold together complexes of interacting genes that together are adapted to components of the environment-that is, there is "coadaptation" (Dobzhansky, 1950). These models, which are in no sense exclusive, are referred to as the "genic" and "karyotypic" hypotheses by Wasserman (1968). He further suggests that if several different coadapted combinations of genes exist within one arrangement in a population, then homozygotes will suffer an extra disadvantage due to disruption of these combinations by recombination. This he calls the "supergenic" level of selection.

Circumstantial evidence from the geographical distribution of inversions, clines, seasonal cycles and long-term frequency changes, tends to support

* Present address: School of Biological Sciences, University of East Anglia, Norwich, NR4 7TJ the karyotypic selection model at least for Drosophila pseudoobscura and persimilis (Dobzhansky, 1970). More direct evidence in favour of coadaptation came from the results of inter-population crosses in $D$ pseudoobscura. Chromosomes of different arrangements from the same population showed heterosis, and reached stable equilibria in population cages. Chromosomes from geographically separated populations did not show heterosis, and the outcome of population cage experiments was unpredictable (Dobzhansky, 1950; Dobzhansky and Pavlovsky, 1953, 1958) although in some cases stable equilibria were reached and heterosis developed (Dobzhansky and Levene, 1951). These results are explicable if the inversions hold together different coadapted combinations of alleles in separate populations, but cannot easily be explained on the genic hypothesis. However, there was the possibility that heterosis was due to an interaction with the genetic background, rather than a property of the inverted region itself, and that the mixed background of the inter-population crosses was the cause of the loss of heterosis. Dobzhansky (1950) excluded this possibility by constructing a cross between Standard and Arrowhead arrangements from Mexico and California of the type: $S T^{M} / A R^{C} \times$ $S T^{C} / A R^{M}$. In the offspring the background was mixed but structural heterozygotes had both chromosomes from the same population. There was heterosis which was, therefore, a result of coadaptation within the inversion. 
Wasserman and Koepfer have taken this approach further by measuring the relative contributions of the genic, karyotypic and supergenic levels of selection on viability and egg hatch, in both Drosophila pseudoobscura and D subobscura (Wasserman, 1968, 1972; Wasserman and Koepfer, 1975). They used crosses between inbred lines from a single population in each case. In $D$ subobscura for the $U_{S T}$ and $U_{1+2}$ arrangements there was a large karyotypic effect, and a small but significant supergenic effect. For the AR and PP arrangements in $D$ pseudoobscura only a karyotypic effect was detected. In neither case was there any evidence for a genic effect.

From a theoretical point of view, it seems likely that some degree of epistasis is necessary for the establishment of a new inversion (Charlesworth and Charlesworth, 1973), but that genic selection is capable of maintaining an inversion polymorphism (Franklin and Lewontin, 1970). Furthermore, some genic contribution is expected to develop as an inversion spreads irrespective of the type of selection involved in its establishment. It is surprising therefore, that there is no experimental evidence of genic selection contributing to the maintenance of an inversion polymorphism. However, the question has only been addressed for Drosophila pseudoobscura and D. subobscura, despite the widespread occurrence of inversion polymorphism in other Drosophila species, and in many other Dipteran families (Dobzhansky, 1970).

We describe here results of an investigation into the selection acting on an inversion polymorphism in the seaweed fly Coelopa frigida (Diptera: Coelopidae). The $\alpha$ and $\beta$ sequences of the first chromosome in $C$. frigida occur at stable frequencies in all populations so far studied in northern Europe (Butlin et al., 1982a), although there may be seasonal (Butlin, 1983) and clinal variation (Day et al., 1982) within very narrow limits. Heterozygotes are nearly always found in excess of Hardy-Weinberg expectations in natural populations and show higher viability than either homozygote under laboratory conditions, especially at high larval densities (Butlin et al, 1984). The karyotypes also differ in egg to adult development time (Day et al., 1980) and adult size (Butlin et al., 1982b). A polymorphism at the alcohol dehydrogenase locus $(A d h)$ is associated with the inversion polymorphism (Day et al., 1982). Of the five known $A d h$ alleles, $A d h-A$ and $A d h-E$ are rare, $A d h-B$ is almost always found on the $\alpha$ sequence and $A d h-D$ on the $\beta$ sequence, while $A d h-C$ may be associated with either sequence.
We have constructed crosses within and between lines derived from widely separated natural populations, and have estimated the genotype frequencies, viabilities and development times in the various sets of progenies. The results point towards a genic rather than a karyotypic basis for heterosis.

\section{MATERIALS AND METHODS}

The general approach was to derive a series of lines from each of two widely separated populations which were isogenic with respect to $A d h$, and thus for most, if not all, of the genes within the inversion. The lines were then crossed both within and between populations to produce $\mathrm{F} 1$ and $\mathrm{F} 2$ generations. The egg to adult viability and, in some cases, the development times of the offspring, were measured.

\section{Derivation of lines}

The lines were derived from collections of larvae made at St Mary's Island, Tyne and Wear (Ordnance Survey grid reference NZ350753) in September 1980, and at Morfa Nefyn, Gwynedd (SH296408) in December 1980. These two populations from the north-east coast of England and the west coast of Wales are separated by several hundred kilometres of coastline including long sections that do not support Coelopa colonies. In each case a series of pair matings was established, and from these were selected crosses of the type $X X \times$ $X Y$ where $Y$ was the required allele at the $A d h$ locus, and $X$ was any allele other than $Y$. The offspring of each cross were sib mated until a line fixed for $Y$ was obtained. This involved between 3 and 8 generations of sib mating (mean 3.95), and produced 16 lines from the St Mary's Island population $(S)$, and 4 from Morfa Nefyn $(M)$. Larvae from the lines were karyotyped from salivary gland smears-the $S$ lines included 11 fixed for the $\beta$ arrangement and 5 for the $\alpha$ arrangement, while 2 of the $M$ lines were fixed for each arrangement.

The mode of derivation of these lines means that they were isogenic for Adh. However, they were not necessarily fixed for other loci within the inversion because some of the $X$ alleles were on the same arrangement as the $Y$ allele and recombination was, therefore, possible. However, all alletes present in a $\beta$ line must have been present on the $\beta$ arrangement in the original population. The lines 
were also inbred to varying degrees and as a result showed some reduction in viability (Butlin 1983).

\section{Procedure for crosses}

Males and virgin females were collected from stock lines and from F1 crosses by standard techniques (Butlin, 1983). They were used to establish the experimental replicates as follows. From one to five pairs in the appropriate combination were left to lay eggs overnight in a plastic canister $(7.5 \mathrm{~cm}$ diameter, $6.5 \mathrm{~cm}$ deep) containing $3 \mathrm{~cm}$ depth of culture medium (Day and Buckley, 1980). The eggs were counted before they hatched and the canister was then kept at $27^{\circ} \mathrm{C}$ in constant darkness. Emerging adults were collected daily and, where necessary, stored at $-20^{\circ} \mathrm{C}$ for subsequent $A d h$ typing. Collection ended when a canister had failed to produce adults on three consecutive days. For offspring of the F2 and FX generations (see below), the karyotypes were deduced from their $A d h$ genotypes. Details of the methods for $A d h$ genotyping are given in Butlin et al., (1982a).

The following crosses were performed:

(a) Fl crosses between lines within the St Mary's Island population in $\alpha \alpha \times \beta \beta$ and $\beta \beta \times \beta \beta$ combinations,

(b) F2 crosses between sibs in the offspring of group (a),

(c) Fl crosses between lines from the St Mary's Island and Morfa Nefyn populations in $\alpha \alpha \times$ $\beta \beta$ and $\beta \beta \times \beta \beta$ combinations, (d) F2 crosses between sibs in the offspring of group (c),

(e) FX crosses between different group (c) sibships of the type $\alpha^{S} \beta^{M} \times \alpha^{M} \beta^{S}$ (analogous to the crosses used by Dobzhansky (1950) to distinguish the effect of a mixed genetic background).

Each cross was replicated between 2 and 6 times and each group contains replicates of several different combinations of lines. The number of eggs differed between replicates but care was taken to ensure a similar distribution of egg numbers within each group of crosses. Numbers ranged from 25 to 450 with the majority in the range 50 to 250 . Hatch rate in $C$. frigida is generally about 98 per cent (Collins, 1978).

\section{RESULTS}

\section{Heterosis in the F2 and FX}

The karyotypic and genic selection hypotheses make clearly distinct predictions about the pattern of relative viabilities in the $\mathrm{F} 2$ and $\mathrm{F} X$ generations. On the karyotypic hypothesis heterosis depends on the coadaptation of chromosomes from the same population. An excess of heterozygotes is expected in the intra-population $\mathrm{F} 2$ and $\mathrm{F} X$ crosses where the $\alpha$ and $\beta$ chromosomes are both derived from one population, but not in the interpopulation F2 crosses where the two chromosomes come from different populations. By contrast, the

$$
\alpha \alpha \quad \alpha \beta \quad \beta \beta
$$

F2 $\alpha \beta$

(Inter- or intra-population)

$d 1+++d 5++$

$d 1+++d 5++$

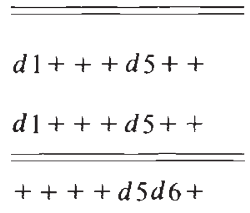

Adh homozygote

F2 $\beta \beta$

$$
\frac{+++d 4++d 7}{+++d 4++d 7}
$$

$$
\begin{aligned}
& ++d 3+t+d 7 \\
& \underline{d 1+++d 5++} \\
& ++d 3+++d 7 \\
& \text { and } \\
& +++d 5 d 6+ \\
& ++d 4++d 7
\end{aligned}
$$

\section{Adh heterozygote}

$$
\frac{+++d 4++d 7}{++d 3+++d 7}
$$

$++d 3+++d 7$

$$
++d 3+++d 7
$$$$
+++d 4++d 7
$$$$
++d 3+++d 7
$$

\section{Adh homozygote}

$$
=\frac{+d 3+++d 7}{++d 3+++d 7}
$$

Figure 1 Explanation of heterozygote excess on the genic hypothesis. Each " $d$ " represents a recessive deleterious allele. $\alpha$ and $\beta$ chromosomes have different sets of deleterious alleles but individual $\beta$ chromosomes share some such alleles irrespective of their population of origin. This predicts a similar, low level of excess in the F2 $\beta \beta$ and FX crosses and a higher level in the F2 $\alpha \beta$ crosses. 
genic hypothesis predicts that relative viabilities will depend on the frequency of homozygosity for recessive deleterious alleles (fig 1). $\alpha$ and $\beta$ chromosomes will have few such alleles in common so that in general $\alpha \beta$ individuals will suffer less homozygosity than $\alpha \alpha$ or $\beta \beta$ individuals. However, where two $\alpha$ (or $\beta$ ) chromosomes are derived from separate populations they too are unlikely to share deleterious alleles. An excess of $\alpha \beta$ heterozygotes is, therefore, expected in the intra- and inter-population F2 crosses but this will be reduced in the $F X$-the only crosses where chromosomal homozygotes have homologues derived from different populations.

When considering viabilities, the expectations are further complicated by the effects of larval competition at different densities. It is useful to separate selection into a component which is not influenced by competition-called "hard" selection (Wallace, 1981) - and a density dependent component of "soft" selection. In principle, either of these components could operate at the karyotypic or the genic level and they can be distinguished in an analysis of the regression of $\alpha \beta$ frequency amongst adults on larval density.

The expectation for these crosses on the hypothesis of karyotypic selection, therefore, was that the $\alpha \beta$ karyotype would be in excess in intrapopulation $\alpha \beta \times \alpha \beta \mathrm{F} 2$ crosses and in the $\mathrm{F} X$ but not in the inter-population F2 crosses. Comparisons between the results of individual replicates and the $1: 2: 1$ expectation (table 1) show the expected tendency towards heterozygote excess in the intra-population crosses, but a similar tendency in inter-population crosses, and a reduced heterozygote excess in the $\mathrm{FX}$-a pattern which conforms to the expectation on the genic hypothesis.

A more critical comparison between the types of cross has been made by analysing the proportion of $\alpha \beta$ individuals amongst the survivors. (This and all subsequent analyses involving proportions use the data after angular transformation). Allowance could then be made for the known effect of larval density on relative viabilities (Butlin et al., 1984).

Regression equations are given in table 2. Considering the F2 first, the effect of density was much more marked in the intra-than the inter-population crosses. However, the regression of $\alpha \beta$ frequency on density was not significant $\left(F_{1,78}=0.92\right)$ and there were no significant differences between the two types of $F 2$ in either the intercept $\left(F_{1,78}=0 \cdot 81\right)$ or the slope of the line $\left(\mathrm{F}_{1,78} \times 1 \cdot 27, P>0 \cdot 20\right)$. The regression for the $F X$ crosses had a lower intercept than the combined F2 (table 2: $F_{1,1104}=4 \cdot 76, P<$ $0.05)$, but the slopes did not differ significantly $\left(F_{1,104}=0.88\right)$. This confirms that there was no difference between inter- and intra-population F2 crosses, but that the proportion of heterozygotes in the $F X$ crosses was lower than in the F2. In addition, the $\mathrm{F} X$ showed a greater effect of density than the F2 intra-population crosses, and the F2 inter-population crosses showed no significant density effect.

Table 1 Comparison of karyotype frequencies with Mendelian expectations

\begin{tabular}{|c|c|c|c|}
\hline Cross & $\begin{array}{l}\text { No. of replicates in which } \\
\text { proportion of heterozygotes } \\
\text { exceeded } 50 \%\end{array}$ & $\begin{array}{l}\text { No. of replicates in which deviation } \\
\text { from } 1: 2: 1 \text { was significant at } P \ll 0.05\end{array}$ & $\begin{array}{l}\text { Total no. of } \\
\text { replicates }\end{array}$ \\
\hline Intra-population $\mathrm{F} 2$ & $27(75 \%)$ & 8 & 36 \\
\hline Inter-population $\mathrm{F} 2$ & $40(80 \%)$ & 9 & 50 \\
\hline$F X$ & $17(65 \%)$ & 2 & 26 \\
\hline
\end{tabular}

Table 2 Relationship between genotype frequencies and larval density

\begin{tabular}{|c|c|c|}
\hline \multirow[b]{2}{*}{ Cross } & \multicolumn{2}{|c|}{$\begin{array}{l}\text { Regressions of } \alpha \beta \text { frequency among adults (in } \\
\text { angles) on numbers of eggs per canister* }\end{array}$} \\
\hline & Intercept \pm S.E. & Slope S.E. $\left(\times 10^{4}\right)$ \\
\hline Intra-population F2 & $0.538 \pm 0.036, P>0.20$ & $5 \cdot 41 \pm 2 \cdot 50, P<0 \cdot 0.5$ \\
\hline Inter-population F2 & $0.630 \pm 0.042, P<0.05$ & $0.45+2.86, P=0.20$ \\
\hline F2 all replicates & $0.600 \pm 0.030, P<0.05$ & $1.96+2 \cdot 05, P>0 \cdot 20$ \\
\hline $\mathrm{F} X$ & $0.461+0.039, P>0 \cdot 10$ & $8.41 \pm 3.45, P \cdot 0.05$ \\
\hline
\end{tabular}

* The intercept is a measure of heterosis in the absence of competition where a value greater than 0.524 represents an excess of heterozygotes over homozygotes ( $P$ values for deviation from this expectation). The slope measures the effect on heterosis of increasing competition ( $P$ values for deviation from zero). 
The position of the intercept represents the hard, density-independent selection acting on homozygotes relative to heterozygotes. If this selection was karyotypic, then the F $X$ and intrapopulation F2 crosses would show the same intercept. In addition, the inter-population F2 would show a lower intercept, close to 50 per cent $(0 \cdot 524$ on the transformed scale). Alternatively, with genic selection, (see fig. 1) the inter-population F2 should show a slightly higher proportion of heterozygotes than the intra-population F2. This is because structural homozygotes are homozygous for most genes within the inversion in both cases, while inter-population structural heterozygotes will on average be slightly more heterozygous than intra-population $\alpha \beta$ individuals. In the FX, structural homozygotes have one chromosome from each population and so are much less homozygous than in the F2-on the genic hypothesis the FX should show less heterosis. Clearly, so far as hard selection is concerned, the results support the genic rather than the karyotypic mode of selection.

The slopes of the regressions on density represent the soft component of selection. In this case the results are more consistent with the expectations on the karyotypic selection hypothesis. Both the intra-population F2 and the FX crosses show a significant effect of density, while the inter-population F2 crosses do not. However, there are no significant differences among the slopes of the regressions for the three types of cross so that, if a karyotypic effect does exist it is weak compared with the genic effect.

Further evidence for a contribution of genic selection can be obtained from the $\beta \beta \times \beta \beta$ F2 crosses. On the karyotypic hypothesis, there is no reason to expect any difference between inter- and intra-population crosses of this type, or to expect, in either case, an excess of Adh heterozygotes. However, Adh heterozygotes have their two copies of the $\beta$ sequence derived from different lines, while homozygotes have both copies from one line. If there is genic selection, an excess of the $A d h$ heterozygotes is expected, and this may be greater in the inter-population than in the intra-population crosses. In fact, both groups showed a slight, nonsignificant, excess of $A d h$ heterozygotes, but there was no difference between inter- and intra-population crosses. The mean proportion of heterozygotes for the intra-population crosses was $0.550 \pm 0.025$ and for the inter-population crosses was $0.542 \pm$ $0 \cdot 020$.

It may be argued that the heterozygote excess in the $\alpha \beta \mathrm{F} 2$ crosses is due to the fact that the $\alpha \alpha$ and $\beta \beta$ homozygotes in these crosses have both chromosomes derived from the same line, and are thus homozygotes for most loci within the inversion. The heterozygosity within the inversion depends on the frequency of recombination within the inversion during the derivation of a line and on the amount of variation in the base population. However, it will certainly be much less than the variation between lines. In the $\mathrm{F} X$, homozygotes do not have both chromosomes from one line and this might explain the lack of heterozygote excess. If this was the case, and the inversion had no effect on the distribution of deleterious recessive alleles, the $\beta \beta \mathrm{F} 2$ crosses would show the same level of heterozygote excess as the $\alpha \beta \mathrm{F} 2$ crosses because $A d h$ homozygotes in the $\beta \beta$ crosses are homozygotes for most of the loci in the inversion, in the same way as chromosomal homozygotes in the $\alpha \beta$ crosses. In fact, the proportion of heterozygotes is significantly higher in the F2 $\alpha \beta$ than the F2 $\beta \beta$ $\left(\mathrm{F}_{1,107}=6.82, \quad P<0.05\right)$ and does not differ between the $F 2 \beta \beta$ and the $F X\left(F_{1,49}=0 \cdot 34\right)$. This means that two independently derived $\beta$ chromosomes are more likely to share deleterious alleles than a $\beta$ chromosome is to share deleterious alleles with an $\alpha$ chromosome. This is the basis of the genic selection hypothesis (fig. 1). Furthermore, the lack of difference between the $F X$ and F2 $\beta \beta$ confirms the importance of genic relative to karyotypic effects.

\section{Viability of the F1 crosses}

It may also be possible to distinguish genic and karyotypic modes of selection in the $F 1$, although in this case the picture may be confused by effects on viability of loci outside the inversion, including coadaptation of the background genotypes of the two populations (Hedrick et al., 1978). The test is also less sensitive because the comparison is between viabilities in different monocultures, rather than between genotypes in the same segregating culture. Nevertheless, the karyotypic mode of selection would predict that the intrapopulation heterozygotes would have higher viability than the inter-population heterozygotes which would be similar in viability to the homozygotes. In contrast, the genic hypothesis would predict that heterozygotes would have higher viability than homozygotes and that inter-population homozygous crosses would have higher viability than intra-population homozygous crosses.

The mean viabilities are given in table 3 . There was no significant effect of density on viability in the range used. Neither karyotype $\left(F_{1,82}=3 \cdot 36\right.$, $P>0.05)$ nor population $\left(F_{1,82}=0.57\right)$ had any 
Table 3 Proportions of eggs surviving to adulthood (in angles) in the Fl generation

\begin{tabular}{llc}
\hline Karyotype & $\begin{array}{l}\text { Intra-population } \\
(\text { mean } \pm \text { S.E. })\end{array}$ & $\begin{array}{l}\text { Inter-population } \\
(\text { mean } \pm \text { S.E. })\end{array}$ \\
\hline$\alpha \beta$ & $0.636 \pm 0.075$ & $0.513 \pm 0.046$ \\
$\beta \beta$ & $0.649 \pm 0.072$ & $0.775 \pm 0.116$ \\
\hline
\end{tabular}

significant effect and there was no significant interaction $\left(F_{1,82}=2 \cdot 47, P>0 \cdot 10\right)$. The directions of the differences do not support either model consistently. The large errors on these mean viabilities suggest that chance effects on viability are too great to detect the genetic effects in simple monocultures of this type.

\section{Development times}

In the laboratory, $\alpha \alpha$ and $\alpha \beta$ males have longer development times than $\beta \beta$ males, and there is little difference between females. The limited evidence available suggests that this is also true of natural populations (Day et al., 1980). Development times of all karyotypes are longer at high larval densities (Butlin and Day, 1984).

The regressions of egg to adult development time (using replicate means on a logarithmic scale) on the initial number of eggs have been examined for the $\alpha \beta \times \alpha \beta$ F2 crosses (table 4). Within the inter- and intra-population groups the pattern was similar. The slopes-that is the effect of density on development time-did not differ between sexes or karyotypes, but the $\alpha \alpha$ and $\alpha \beta$ males had consistently longer development times than the $\beta \beta$ males and females of all karyotypes. This confirms previous observations (Butlin and Day, 1984), and suggests that the two populations do not differ markedly in the effects of the chromosomal arrangements on development time. However, while the inter- and intra-population groups did not differ in their intercepts, the slopes for the inter-population group were always less than for the intra-population group $(P<0.05$, sign test $)$.

This difference is consistent with the explanation proposed for the heterozygote excess in interand intra-population crosses. It was suggested that the density independent advantage to heterozygotes in the inter-population crosses was due to genic effects-probably homozygosity for deleterious recessives in the structural homozygotes. This selective mortality may well be around the time of hatching. If so, it would have the effect of reducing larval density in the later instars where most of the effect on development time is experienced. Conversely, in the intra-population crosses there was less density-independent mortality, and more density-dependent mortality. The latter probably results from competition between the karyotypes, with the coadapted heterozygote being at an advantage. Competition would produce mortality late in larval life or in the pupal stage, and so would not reduce the effective larval density with respect to development time. Thus the interpopulation crosses are expected to show shorter development times than the intra-population crosses, especially at high densities. This is the observed pattern.

In summary, the measurements of heterosis in the $\mathrm{F} 2$ and $\mathrm{FX}$ crosses strongly indicate that a

Table 4 Density and Development Time in the F2

\begin{tabular}{|c|c|c|c|c|}
\hline \multirow[b]{2}{*}{ Cross } & \multirow[b]{2}{*}{ Sex } & \multirow[b]{2}{*}{ Karyotype } & \multicolumn{2}{|c|}{$\begin{array}{l}\text { Regression of replicate mean development } \\
\text { time (log days) on initial no. of eggs }\end{array}$} \\
\hline & & & Intercept $\pm S . E$ & Slope \pm S.E. $\left(\times 10^{4}\right)$ \\
\hline \multirow[t]{6}{*}{ Intra-population } & Male & $\alpha \alpha \psi$ & $1 \cdot 17 \pm 0.04$ & $13 \cdot 38 \pm 3 \cdot 77^{* * *}$ \\
\hline & & $\alpha \beta$ & $1 \cdot 15+0 \cdot 04$ & $9 \cdot 81+3 \cdot 79^{*}$ \\
\hline & & $\beta \beta$ & $1 \cdot 06 \pm 0 \cdot(13$ & $9 \cdot 94 \pm 3 \cdot 21^{* *}$ \\
\hline & Female & $\alpha x<Y$ & $1 \cdot 11+0 \cdot 04$ & $12 \cdot 36 \pm 3 \cdot 62^{* * *}$ \\
\hline & & $\alpha \beta$ & $1.06 \pm 0.04$ & $12 \cdot 80+3 \cdot 56^{* * *}$ \\
\hline & & $\beta \beta$ & $1 \cdot 06 \pm 0 \cdot 03$ & $12 \cdot 05+3 \cdot 29 * *$ \\
\hline \multirow[t]{6}{*}{ Inter-population } & Male & $\alpha<\psi$ & $1 \cdot 18 \pm 0 \cdot 02$ & $7 \cdot 55 \pm 2 \cdot 15^{* *}$ \\
\hline & & $\alpha \beta$ & $1 \cdot 17+0 \cdot 02$ & $4 \cdot 62+1 \cdot 83^{*}$ \\
\hline & & $\beta \beta$ & $1 \cdot 09 \pm 0 \cdot 02$ & $4 \cdot 81 \pm 1.77^{* *}$ \\
\hline & Female & $\alpha x<$ & $1 \cdot 06 \pm 0 \cdot() 3$ & $8 \cdot 21 \pm 2 \cdot 54^{* *}$ \\
\hline & & $\alpha \beta$ & $1.07+0.02$ & $7 \cdot 90 \pm 2 \cdot 06^{* * * *}$ \\
\hline & & $\beta \beta$ & $1 \cdot 10+0 \cdot 02$ & $5 \cdot 76+1 \cdot 94^{* *}$ \\
\hline
\end{tabular}

Significance levels: * $P<0 \cdot 05, * * P<0 \cdot 01,{ }^{* * *} P<0 \cdot(0) 1$. 
major part of selection is operating at the genic level. The results from the Fl crosses do not allow us to reach any conclusion, but the analysis of development times supports the interpretation of density-independent genic selection and densitydependent karyotypic selection.

\section{DISCUSSION}

Individuals heterozygous for the two arrangements of chromosome I in Coelopa frigida have higher viability than either homozygote both in the laboratory and in the field (Butlin et al., 1982a, 1984). This is probably the major selective force maintaining the polymorphism, although inversion frequencies are also influenced by sexual selection, and selection on development time (Day et al., 1980, Butlin et al., 1982b). The results presented here suggest that the viability selection is principally due to genic, rather than karyotypic, selection. This is a density-independent effect due to homozygosity for recessive deleterious alleles. There may also be a density-dependent component due to coadaptation of genes within the inverted region. The latter results in heterozygotes that are better competitors than either homozygote. This interpretation is consistent with the earlier observations that, on the one hand, there is some heterozygote excess even at very low, non-competitive, larval densities, and on the other, that the excess is greater under conditions of intense larval competition (Butlin et al., 1984).

A mixture of karyotypic and genic modes of selection is to be expected for an inversion polymorphism. Epistatic interactions are necessary for the establishment of a new inversion, but once established, an inversion will accumulate deleterious recessive alleles (Charlesworth and Charlesworth, 1973). It is surprising then that this seems to be the first report of a significant contribution of genic selection. The reason may be that in order to detect the contributions of genic and karyotypic selection to viability, it was necessary to examine the relationship between viability and larval density. Previous experiments have generally used only a single larval density.

It is also important to recognise that viability selection is just one component of the selection on an inversion polymorphism. Most inversions have many and varied phenotypic effects, and are subject to selection in many ways (Dobzhansky, 1970, 1971). It is essential to study the contributions of genic and karyotypic effects to selection on a wider range of characters and in a wider range of species before making generalisations about the way in which inversion polymorphisms are maintained.

Acknowledgements The support of the SERC to RKB is gratefully acknowledged.

\section{REFERENCES}

BUTLIN, R. K. 1983. The maintenance of an inversion polymorphism in Coelopa frigida. Ph.D. Thesis, University of Nottingham.

BUTLIN, R. K. AND DAY, T. H. 1984. The effect of larval competition on development time and adult size in the seaweed fly, Coelopa frigida. Oecologia, 63, 122-127.

BUTLIN, R. K., COLLINS. P. M. AND DAY, T. H, 1984. The effect of larval density on an inversion polymorphism in the seaweed fly, Coelopa frigida. Heredity, 52, 415-423.

BUTLIN, R. K., COLLINS, P. M., SKEVINGTON, S. J. ANIJ DAY, T. H. $1982 a$. Genetic variation at the alcohol dehydrogenase locus in natural populations of the seaweed fly, Coelopa frigida. Heredity, 48, 45-55.

BUTLIN, R. K., READ, I. L. AND DAY. T. H. 1982 $b$. The effects of a chromosomal inversion on adult size and male mating success in the seaweed fly, Coelopa frigida. Heredity, 49, $5 \mathrm{I}-62$.

CHARLESWORTH, B. AND CHARLESWORTH, D. 1973. Selection of new inversions in inulti-locus genetic systems. Genetical Research, 21, 167-183.

COLLINS, P. M. 1978. Studies on genetic polymorphism in Coelopa frigida. Ph.D. Thesis, University of Nottingham.

DAY, T. H. AND BUCKLEY, P. A. 1980. Alcohol dehydrogenase polymorphism in the seaweed fly, Coelopa frigida. Biochem. Genet., 18, 727-742

IDAY, T. H., DOBSON, T., HILLIER, P. C., PARKIN, D. T. ANI) CLARKF., B. 1980. Different rates of development associated with the alcohol dehydrogenase locus in the seaweed fly, Coelopa frigida. Heredity, 44, 321-326.

DAY, T. H., DOBSON, T., HILLIER, P. C., PARKIN, D. T. ANI CLARKE, B. 1982. Associations of enzymic and chromosomal polymorphisms in the seaweed fly, Coelopa frigida. Heredity, 48, 35-44

DOBZHANSKY, T. 1950. Genetics of natural populations XIX: Origin of heterosis through natural selection in populations of Drosophila pseudoobscura. Genetics, 35, 288-302.

DOBZHANSKY, T. 1970. The Genetics of the Evolutionary Process. Columbia University Press, New York.

DOBZHANSKY, T. 1971. Evolutionary oscillations in Drosophila pseudoobscura. R. Creed, (ed.) In Ecological Genetics and Evolution, pp 109-133. Blackwell Scientific Publications, Oxford.

DOBZHANSKY, T. AND LHVF.NE, H. 1951. Development of heterosis through natural selection in experimental populations of Drosophila pseudoobscura. American Naturalist, 85 $247-264$

DOBZHANSKY, T. AND PAVLOVSKY, O. 1953. Indeterminate outcome of certain experiments on Drosophila pseudoobscura. Evolution, 7, 198-210.

DOBZHANSKY, T. AND PAVLOVSKY, O. 1958. Interracial hybridization and breakdown of coadapted gene complexes in Drosophila paulistorum and Drosophila willistoni. Proceedings of the National Academy of Sciences, U.S.A $44,622-629$ 
FRANKLIN, I. AND LEWONTIN, R. C. 1970. Is the gene the unit of selection? Genetics, 65, 707-734.

HEDRICK, P., IAIN, S. AND HOLDFN, 1. 1978. Multilocus systems in evolution. Evolutionary Biology, 11, 104-184.

OHTA, T. 1971. Associative overdominance caused by linked detrimental mutations. Genetical Research, 18, 227-286.

STURTEVANT, A. H. AND MATHER, K. 1938. The interrelations of inversions, heterosis and recombination. American Naturalist, 72, 447-452. wallace, B. 1981. Basic Population Genetics. Columbia University Press, New York.

WASSERMAN, M. 1968. Recombination-induced chromosomal heterosis. Genetics, 58, 125-139.

WASSERMAN, M. 1972. Factors influencing fitness in chromosomal strains of Drosophila subobscura. Genetics, 72, 691708.

WASSERMAN, M. AND KOEPFER, H. R. 1975. Fitness of karyotypes in Drosophila pseudoobscura. Genetics, 79, 113-126. 